\title{
Nutrient intake and anthropometrics of vegetarian, vegan and omnivorous children (1-3 y) in Germany
}

\author{
Stine Weder ${ }^{1,2}$, Morwenna Hoffmann ${ }^{1}$, Katja Becker $^{3}$, Ute Alexy ${ }^{4}$ and Markus Keller ${ }^{5}$ \\ ${ }^{1}$ Fachhochschule des Mittelstands, Bielefeld, Germany, \\ ${ }^{2}$ Institute for Alternative and Sustainable Nutrition, Biebertal, Germany, \\ ${ }^{3}$ Justus Liebig University, Giessen, Germany, \\ ${ }^{4}$ University of Bonn, Bonn, Germany and \\ ${ }^{5}$ Fachhochschule des Mittelstands, Cologne, Germany
}

\begin{abstract}
Introduction: Vegetarian (VG) and vegan (VN) diets have become more and more popular in the last decades. Many VG and VN parents raise their children without meat or foods of animal origin. Until now, only few studies - that are highly heterogeneous, quite old and of small sample sizes - have been conducted with VG and VN children worldwide.
\end{abstract}

Materials and methods: The Vegetarian and Vegan Children Study (VeChi Diet Study) collected data of 139 VN, 127 VG, and 164 omnivourous (OM) children (1-3 y) in Germany. Dietary intake (including supplements and fortified foods) was assessed by a 3-dayweighted dietary record; lifestyle and anthropometric data by an online questionnaire. Average dietary intakes and anthropometrics were compared between groups using analysis of covariance (ANCOVA).

Results: Intake of total energy, energy density, retinol eq, phosphorous, and zinc did not significantly differ between the study groups after adjustment. OM children had the highest adjusted intakes of protein, fat, added sugars, vitamin $\mathrm{B}_{2}$, and calcium (the latter only after excluding outliers) $(\mathrm{p}<0.0001)$. In contrast, the highest adjusted intakes of carbohydrates, fiber, vitamin $\mathrm{E}$, vitamin $\mathrm{B}_{6}$, folate, vitamin $\mathrm{C}$, vitamin $\mathrm{K}$, magnesium, and iron were found in VN children ( $\mathrm{p}<0.0001)$. Vitamin $\mathrm{B}_{12}$ supplements were used by $97.1 \%$ of VN, 35.4\% of VG, and 7.9\% of OM children. However, 58 VG (45.7\%), 27 OM (16.5\%), and 14 VN (10.1\%) did not meet the German DRI for vitamin $\mathrm{B}_{12}$. Although there were no differences in the Standard-Deviation-Scores for weight-for-height, height-for-age, or weight-for-age on average (WHO Growth Standards 2011), 3.6\% and 2.4\% of VN and VG children, respectively, were classificated as stunted or severely stunted.

Discussion/Conclusion: Our results indicate that a VG and VN diet in early childhood provides comparable amounts of energy, a macronutrient pattern in accordance with recommendations, and can ensure normal growth as there were no significant differences in proxy-reported anthropometrics compared to OM children of the same age. However, the observed small percentage of VG and VN children in our sample classified as stunted should emphasize to parents and pediatricians the importance of an adequate energy and nutrient intake for children on VG and VN diets. Qualified nutrition counselling for VG and VN children might be useful to ensure a healthy development.

\section{Conflict of Interest}

Stine Weder, Morwenna Hoffmann, Katja Becker, and Ute Alexy declare no conflict of interest. Markus Keller is an unsalaried member of the Scientific Advisory Board of ProVeg Germany. 